CARTA AL DIRECTOR

\title{
Tres exposiciones y un episodio de reexposición de angioedema producido en tratamiento con risperidona
}

\author{
B. Kores Plesnicar, S. Vitorovic, B. Zalar, M. Tomori \\ Clínica Psiquiátrica Universitaria de Ljubljana, Studenec 48, 1260 Ljubljana Polje, Eslovenia
}

En las publicaciones encontramos sólo algunos casos de reacciones cutáneas intensas al medicamento antipsicótico risperidona [2, 4], como monoterapia o en combinación con otros medicamentos psicotropos, sobre todo con valproato $[1,3]$. Nuestro informe describe tres apariciones de edema facial y periorbital con terapia de risperidona en una paciente que se desvanecieron cada vez que el tratamiento con este fármaco se interrumpió.

La Sra. R, una paciente de 63 años con esquizofrenia paranoide crónica, ha estado ingresada en nuestro hospital psiquiátrico durante 36 años. La mayor parte del tiempo su conducta ha sido desorganizada, con alucinaciones auditivas imperativas, arrebatos de agitación intensa y agresión física y verbal. Durante su prolongada hospitalización, ha recibido casi todos los antipsicóticos conocidos, así como clozapina y olanzapina. En 1998, se añadieron $2 \mathrm{mg}$ de risperidona diaria, ajustada a $6 \mathrm{mg}$ diarios en 2 semanas, que se añadieron a flufenacina, biperideno y bromacepam, que, en esa época, la paciente llevaba recibiendo muchos meses. Después de 1 mes, se produjo edema periorbital, que se desvaneció en una semana tras la interrupción del tratamiento con risperidona. Todos los demás medicamentos se mantuvieron. Los valores de laboratorio bioquímicos, hematológicos y tiroideos estaban dentro de lo normal. A causa del deterioro de su estado físico y su falta de respuesta al tratamiento, un año después se introdujo de nuevo risperidona ajustada a $6 \mathrm{mg}$ diarios en 2 semanas en un tratamiento con prometacina, biperideno, clonacepam y nitracepam. Después de un tratamiento de 45 días junto con la risperidona, se produjo edema periorbital y orbital moderado y, como resultado, se interrumpió la prometacina; sin embargo, el edema se mantuvo igual. Cinco días después se interrumpió (también) la risperidona y el edema se desvaneció en 3 días sin ningún tratamiento adicional. Cinco meses más tarde se introdujeron $3 \mathrm{mg}$ diarios de risperidona en un tratamiento ya existente con flufenacina, trihexifenidilo, loracepam y nitracepam. Después de 3 semanas de tratamiento con risperido$\mathrm{na}$, se produjo repentinamente angioedema, que afectó a los labios, el rostro, el cuello y la lengua, amenazando la potencia laríngea. A causa de una hinchazón intensa de la lengua y el estrechamiento de la laringe, la paciente recibió terapia antialergénica intensiva. La risperidona se interrumpió de inmediato. Después de unas horas, el edema disminuyó y la respiración se hizo más fácil; el edema desapareció completamente en 4 días.

Los componentes inmunológicos se midieron únicamente en la última aparición del angioedema. El nivel de C4 se redujo a $131 \mathrm{mg} / \mathrm{L}$ (intervalo normal $138-435 \mathrm{mg} / \mathrm{L}$ ) y la vía clásica total de activación del complemento cayó al $26 \%$ (intervalo normal: $72-128 \%$ ). Los valores de IgE estaban dentro del intervalo normal. La probabilidad de la reacción alérgica de tipo IV era mayor que la de

Kores Plesnicar B, Vitorovic S, B. Zalar B, Tomori M. Three challenges and a rechallenge episode of angio-oedema occurring in treatment with risperidone. Eur Psychiatry 2001; 16: 506-7. 
tipo I. Sin embargo, no se pudo descartar, ya que el curso de la reacción alérgica de tipo I no incluye necesariamente la alteración de los valores de IgE. La triple exposición y reexposición del angioedema con el tratamiento de risperidona confirman el papel de este medicamento en la presencia del angioedema; sin embargo, su mecanismo es difícil de esclarecer. La combinación de medicamentos puede tener cierta significación: en nuestro caso, las benzodiacepinas eran parte ya del tratamiento básico. Los informes adicionales sobre esta materia son bienvenidos.

\section{BIBLIOGRAFÍA}

1 Baldassano C, Nassir Ghaemi S. Generalized edema with risperidone: divalproex sodium treatment [letter]. J Clin Psychiatry 1996; 57: 422.

2 Cooney C, Nagy A. Angio-oedema associated with risperidone [letter]. BMJ 1995; 311: 1204.

3 Sanders RD, Lehrer DS. Edema associated with addition of risperidone to valproate treatment [letter]. J Clin Psychiatry 1998; 59: 689-90.

4 Terao $T$, Kojima $H$, Eto $A$. Risperidone and allergic reactions [letter]. J Clin Psychiatry 1998; 59: 82-3. 